\title{
VISUAL PERCEPTION BASED AUTOMATIC RECOGNITION OF CELL MOSAICS IN HUMAN CORNEAL ENDOTHELIUM MICROSCOPY IMAGES
}

\author{
YANN GAVET AND JEAN-ChaRles PinOli
}

Centre Ingénierie et Santé; LPMG, UMR CNRS 5148; École Nationale Supérieure des Mines de Saint-Etienne, 158 Cours Fauriel, 42023 Saint-Etienne cedex, France

e-mail: gavet@emse.fr

(Accepted January 8, 2008)

\begin{abstract}
The human corneal endothelium can be observed with two types of microscopes: classical optical microscope for ex-vivo imaging, and specular optical microscope for in-vivo imaging. The quality of the cornea is correlated to the endothelial cell density and morphometry. Automatic methods to analyze the human corneal endothelium images are still not totally efficient. Image analysis methods that focus only on cell contours do not give good results in presence of noise and of bad conditions of acquisition. More elaborated methods introduce regional informations in order to perform the cell contours completion, thus implementing the duality contour-region. Their good performance can be explained by their connections with several basic principles of human visual perception (Gestalt Theory and Marr's computational theory).
\end{abstract}

Keywords: cell detection, contour closure, contour-region duality, human corneal endothelium, mosaic reconstruction, specular microscopy.

\section{INTRODUCTION}

\section{CORNEA: VISION AND QUALITY CONTROL}

The cornea is the transparent surface in the front side of the eye. It has a role of protection of the eye. Together with the lens, it enables to focus the incident light onto the retina.

It is constituted of several layers, such as the epithelium (at the frontside of the cornea), the stroma and the endothelium (at the backside of the cornea). The endothelium contains non-regenerative cells tiled in a monolayer and hexagonal mosaic.

This endothelial layer pumps water from the cornea, keeping it clear. A high cell density and a regular morphometry of the cells of this layer reflect the good quality of a cornea before transplantation, the most common medical transplantation in the world. Herein lays the importance of the endothelial quality control.

Before transplantation, the cornea button is observed by classical optical microscopy (ex vivo, Fig. 1). After grafting, the cornea is observed in vivo with an optical specular microscope (Gain et al., 2002; Fig. 2). Those two images have similarities (the cell borders correspond to the intersticial zone that surround the cell bodies): a human observer, expert or not, can easily count the cells and draw their borders.

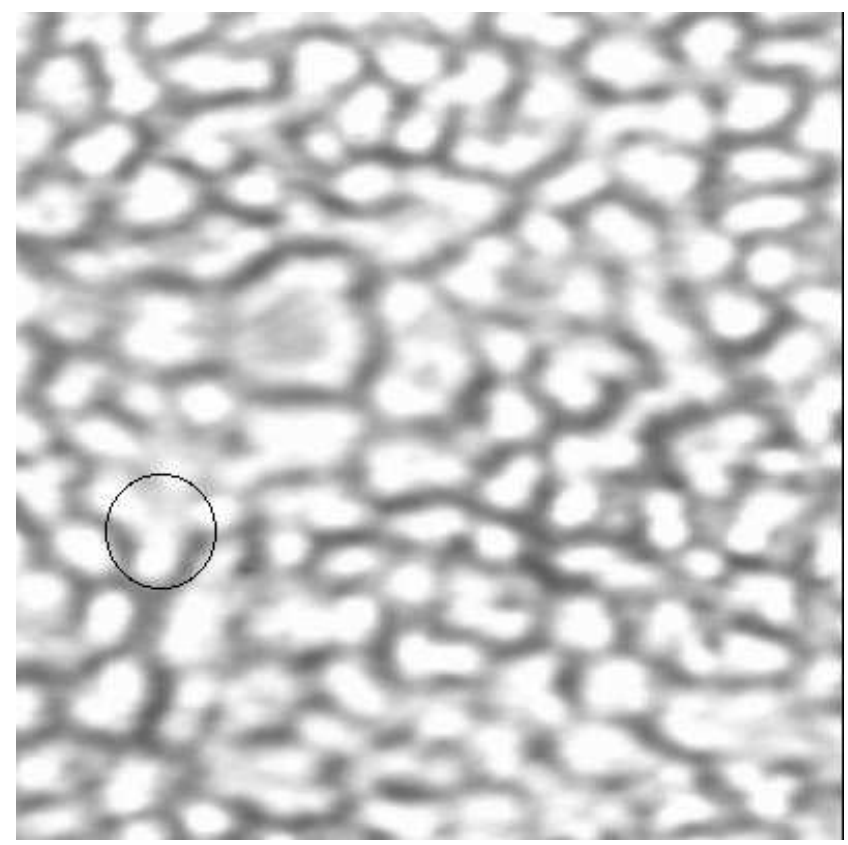

Fig. 1. Optical microscopy imaging of a human corneal endothelium.This image is $400 \times 400$ pixels, representing $0.52 \times 0.52 \mathrm{~mm}$. Within the black circle, one can notice that the endothelial cell borders are not visible and that a lot of informations is lost partly due to the saturation effect. A simple thresholding on this image will thus not give a good segmentation of the cells.

But, due to a lack of informations in some parts of the borders, computer algorithms cannot easily perform the completion of the cell borders. This problem is very time consuming for experts that have to manually take 
the place of the software. Moreover, this operation is simple (for not too bad images) and a child could easily do it visually (but coding it in a computer program is not so easy!).

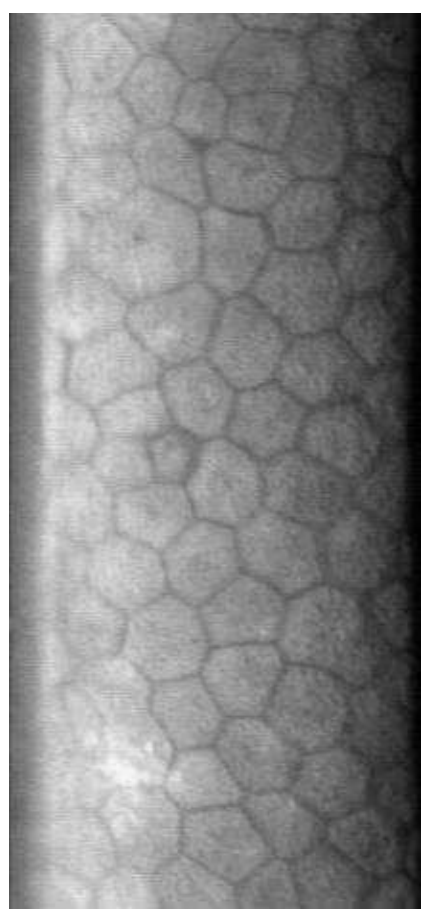

Fig. 2. Specular microscopy imaging of a human corneal endothelium. This 8-bits (256 grey levels) image is constituted by $215 \times 468$ pixels, and represents a spatial field of $0.08 \mathrm{~mm}^{2}(0.19 \mathrm{~mm} \times$ $0.42 \mathrm{~mm}$ ).

\section{THE LIMITS OF EXISTING METHODS}

Several methods exist to evaluate the cell density of the human corneal endothelium. Some perform a global analysis like Doughty et al. (1997); Fitzke et al. (1997), or Ayala et al. (2001). However, these techniques avoid the problem of detecting the cells since they are only focusing on an average cell density computation. Some other methods try to mimic the human visual perception by detecting the borders of the cells (Gain et al., 2002; de Andrade, 2004; Debayle et al., 2006), or by extracting the cell bodies (Foracchia and Ruggeri, 2000).

The first limitation of such methods is that a specialist of the domain still has to interact with the software to either choose a threshold value (Gain et al., 2002), select the center of the cells (de Andrade, 2004) or correct and add borders to the detected ones (Foracchia and Ruggeri, 2000). Second, the algorithm of Gain et al. (2002) is too simple to work well in the case of a poor acquisition step or of bad conditions of lighting (Fig. 1). In specular microscopy, the Fig. 2 presents an illumination deviation (due to the optics of the microscope) that forbids the use of a global threshold to detect the borders of the cells.

Those borders lead into a partition of the plane, i.e., a tesselation that will be called in this article "mosaic", word taken from the ophtalmology vocabulary to describe the pattern formed by the hexagonally-shaped cellular structure of the corneal endothelium.

\section{GOAL OF THIS PAPER}

The goal of this article is to show how we can mimic the visual perception system to computationnaly get the borders of the cornea cells, and to show why some advanced image analysis techniques that give good results in fact involve some vision principles.

\section{IMAGE ANALYSIS TOOLS AND LINKS TO HUMAN VISUAL PERCEPTION}

\section{GESTALT THEORY AND ALGORITHMS}

It is a fact that the human visual perception system is very performant. It does not need complete informations to infer objects (Fig. 3; Kanizsa, 1980) and to understand a scene. Moreover, it has been proved that animals could also percept such subjective figure (Hateren et al., 1990; Nieder, 2002).
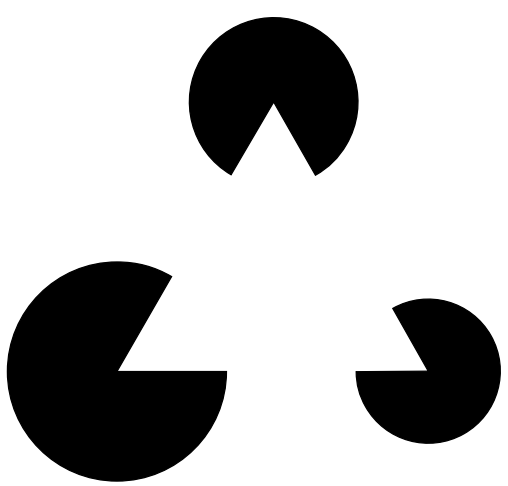

Fig. 3. Kanisza triangle: although not drawn, the triangle can be easily visually infered.

To describe the human visual perception, Wertheimer provided some principles under its Gestalt Theory (Wertheimer, 1923a;b; Koffka, 1935; Rock, 2001). According to it, basic elements like dots, lines and segments, curves, are grouped together according to some visual principles, e.g., continuation (Fig. 4), proximity (Fig. 7) and symmetry. 


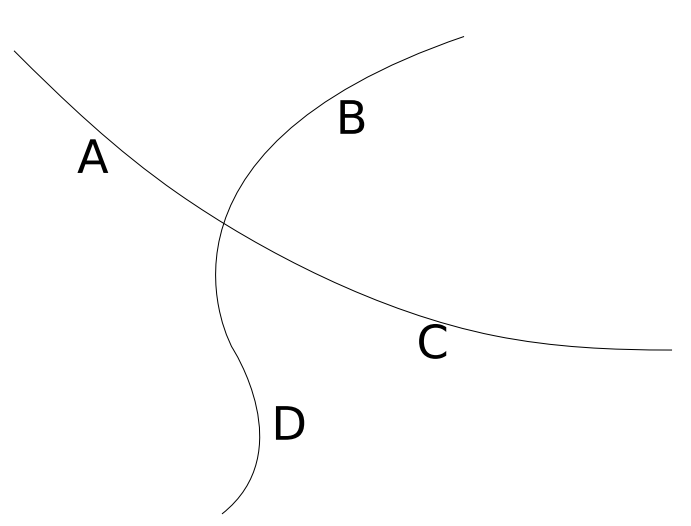

Fig. 4. The lines $A-C$ and $B-D$ are visually grouped by good continuation. On contrary, we have to do an effort to percept the groups $A-B$ and $C-D$.

The continuity of direction between (for example) segments plays an important role. The Gestalt Theory calls it the "good continuation" principle. An angular variation of $30^{\circ}$ has been proved to be a visual "limit" between continuous and intersection lines (Dakin and Hess, 1999; Mullen et al., 2000). Visually, this principle means that a line does not need to be continuously drawn as long as the "good continuation" is respected (Kovács and Julesz, 1993). The implementation of this principle in a computer program consists in testing every pair of segments and to verify if the angle criterion is satisfied or not (see Fig. 5 and Fig. 6).

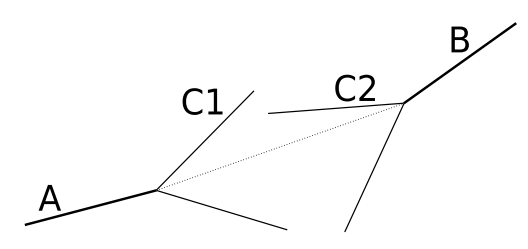

Fig. 5. If the angle between segment $A$ and segment $B$ is less than $30^{\circ}$, then the "good continuation principle" applies between $A$ and $B$ and the continuation line between $A$ and $B$ can be drawn. Notice that this relation is symmetric: $A$ is in the continuation of $B$, as well as $B$ is in the continuation of $A$.

As claimed by the Gestalt Theory, the human visual system groups objects by following the "proximity" principle. Kubovy and Gepshtein (2000) proved, with what they called "the pure distance law", that the direction of an alignment was preferentialy chosen according to the proximity between stimuli. To test the "proximity" principle, the Euclidean distance is used.

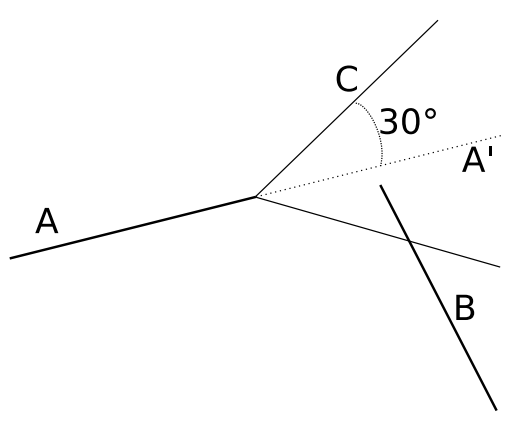

Fig. 6. When the extremity of the segment $B$ lays in the cone $C$, then $B$ is considered as a "weak continuation" of segment $A\left(A^{\prime}\right.$ shows the direction of $\left.A\right)$. The "good continuation" is more restrictive than the weak continuation (see Fig. 5).

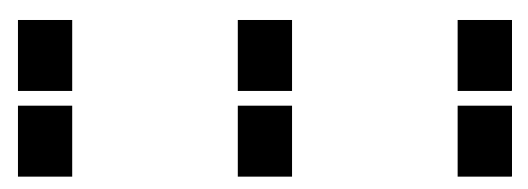

Fig. 7. The six rectangles form 3 groups by "proximity".

\section{SKELETONS, WATERSHEDS AND DISTANCE MAPS}

"Symmetric" objects are visually more important than the others. A symmetry gives informations on the structure of the object. This can be linked to the medial axis (Calabi and Hartnett, 1968; Blum and Nagel, 1978; Zou et al., 2001, and Fig. 8) that concentrates informations about the original shape. Therefore, the hexagonally-shaped structure of the cornea endothelium is very symmetric, and those properties should give practical information to properly detect the cells.

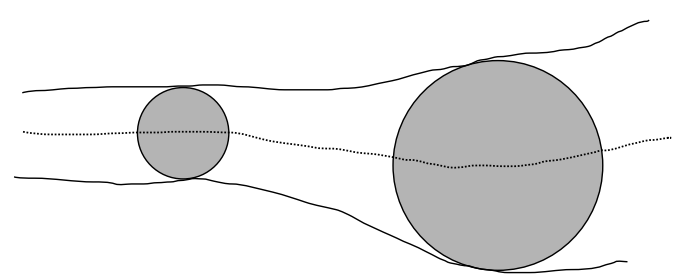

Fig. 8. Illustration of the medial axis, here in dot lines, that summarize the informations of the contours (the connected lines). The medial axis corresponds to the crest line of the distance transform obtained from the contours.

The primal sketch of Marr (1983) is the knowledge of contours. This is the first step in perception according to Marr. When trying to mimic the 
human visual perception system, it seems thus logic to begin with a contour detection, followed by a morphological skeletonization (Soille, 2003), as illustrated in Fig. $11 \mathrm{~b}$.

Closed and convex contours are important to human visual perception (Kovács and Julesz, 1993), and the use of the watershed operator is indeed justified (Beucher and Lantuejoul, 1979). Moreover, a distance map (Cuisenaire, 1999) and its maxima add a regional information that can complete or close the contours (distance map calculated from a watershed or from a skeleton), thus introducing the duality contour-region, which is what was suggested by Marr (1983) for the human visual system: a "top-down-bottom-up" analysis.

\section{BORDERS DETECTION AND RECONSTRUCTION}

The first subsection will show how to detect the borders of the cells. The next subsections will present methods to reconstruct the cell mosaic: first by focusing only on the borders, and then by introducing some regional informations.

\section{SIMPLE METHOD: CELL BORDERS DETECTION}

The generic method for the recognition of the cell mosaic in human corneal endothelium microscopy images is presented through a synoptical scheme (Alg. 1).

The Alg. 1 is a summary of what will be presented in the next subsections. The image filtering and borders detection steps correspond to what a computer can 'see' or not (see Figs. 1, 2): sometimes, the cell borders are not complete. The human visual perception system infers the missing information: this third step is done with a computer program by what is called "reconstruction".

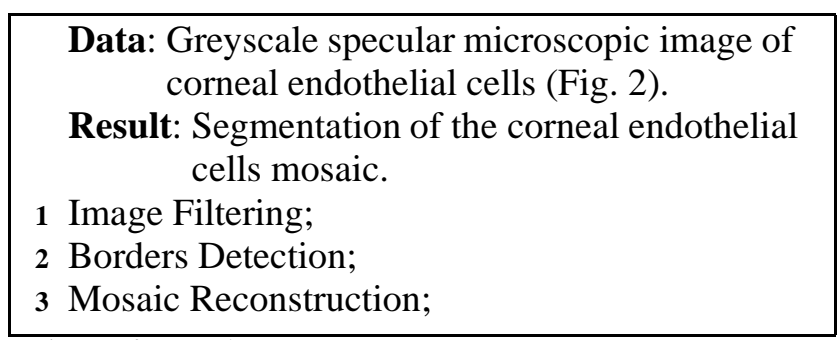

Algorithm 1: General method for detecting and reconstructing the endothelium mosaic.

Indeed, a filtering process is necessary for detecting the borders (see algorithms 2, 3 and 4 below).
This is what is implicitely done by the visual system. The medial axis gives a thin version of the cell borders (Fig. 11b), that we will call cell contours. This skeleton is the dual operation of the SKIZ (skeleton by influence zone, Soille, 2003) that will be performed later.

In the field of mathematical morphology, alternate sequential filters are often used. They consist of an alternance of openings and closings of growing sizes. A more classical filter could also be used (like mean or gaussian filter), but the morphological filter preserves better the borders of the cells.

Neural networks have also been used to perform the task of segmenting corneal endothelium images, as for example in Salerno et al. (1998), where the segmentation into cell bodies and cell boundaries is achieved by a shift invariant artificial neural networks (with the classical backpropagation technique as training process). The drawbacks of this method is the use of a fixed-size mask; notice that an expert is required to perform the necessary corrections, whereas our method intent to compute them automatically.

\section{CONTOUR COMPLETION BY GOOD CONTINUATION}

This first method to reconstruct corneal mosaics is based on the "good continuation" principle from the Gestalt Theory. The angle criterion (see Figs. 5, 6) can easily be applied between line segments. This is why the mosaic is approximated by segments, for example with gestalt based methods, e.g., Hu and Yan (1997).

\section{Synthetical testing mosaic}

To simulate the reconstruction, a synthetical binary mosaic (Fig. 9) is manually drawn. Holes are performed on it and an algorithm tries to reconstruct it. This algorithm is a strict implementation of the "good continuation": every segment can potentially be continued to another one and every pair of segment is thus tested.

The main drawback of the "good continuation" algorithm is obvious: no information about the distance between the segments is used in order to avoid (for example) the cross-over of two continuations. In a second version of this algorithm, a condition of distance is introduced: the continuation of two segments can be done only if the hole between them is smaller than the length of both the original segments. This criterion is still not the good one: it seems that a regional information have to be introduced in order to close the borders of the cells. 


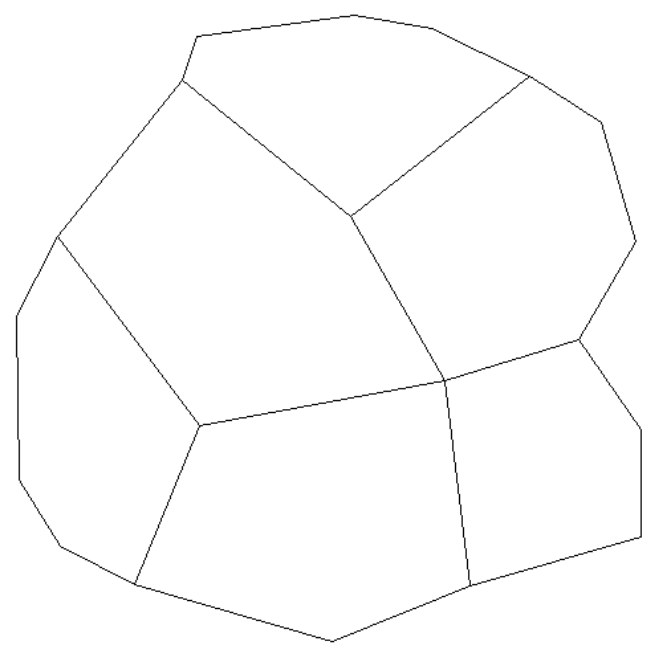

(a)

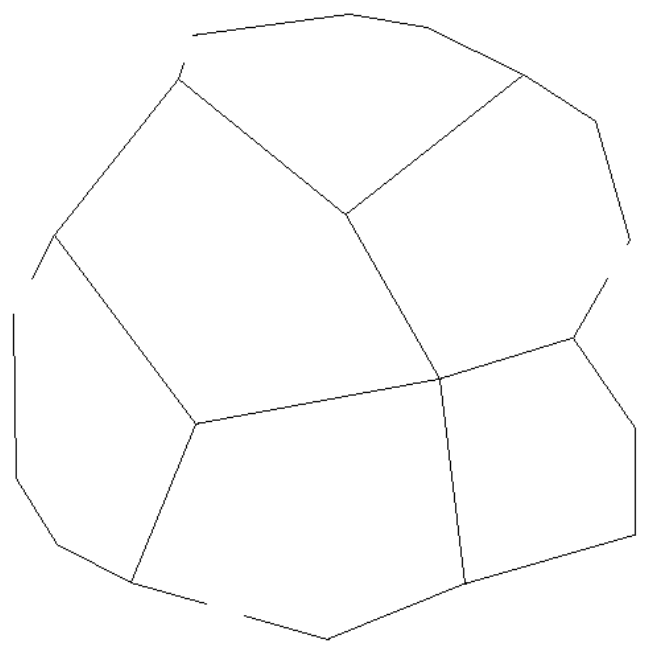

(b)

Fig. 9. A test mosaic and the holes that the "good continuation" algorithm tries to close. (a) A synthetical test mosaic. It will be used as a basic spatial pattern to test the reconstruction methods. (b) Holes are randomly performed into the test mosaic (a) and the "good continuation" algorithm tries to close them. Although this is a synthetical mosaic, the human visual system performs the closing easily.

When using the "good continuation" only (Fig. 10b), the errors come from the fact that there is no information about what couple of extremities are to be reconnected. The distance used in this method is used for comparing "continuations" together, but not to limit the "continuation" to a certain region.

\section{Real cornea mosaic}

Looking at a mosaic over the original cornea gives some more informations about how to close the contours. It seems that there is not a linear path but a geodesic path (if we think about the image as a surface) of "continuation". This can be seen as an extension of the "good continuation principle" to the grey level images. At each extremity of the segments to be continued, the computation of the geodesic shortest path (see Ikonen and Toivanen, 2005) gives a distance value and a path to every other extremity in the mosaic (Fig. 10). The angle criterion is no more used; instead, we use the minimal distance from an extremity to another.

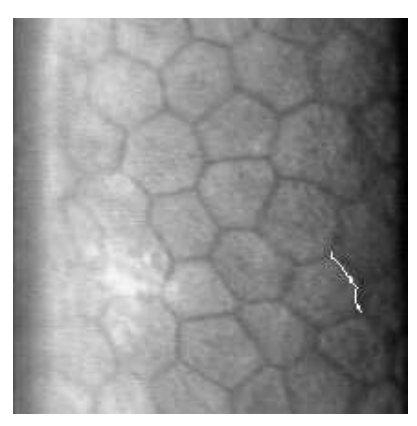

(a)

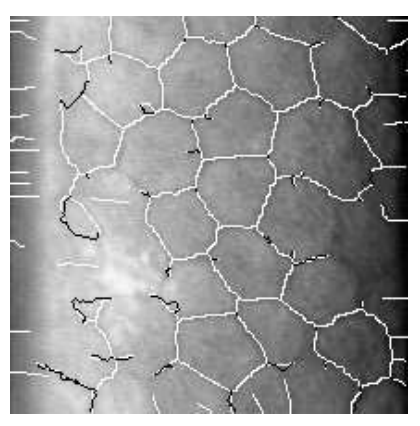

(b)
Fig. 10. The geodesic distance is tested on a subimage extracted from Fig. 2, because it presents typical situations (informations not present but inferred by a human observer). (a) Given two points (the extremities of the white path), the geodesic shortest path between the two points is highlighted here in white. This path is visually following the contours of the cells. It could be used to close the contours of the mosaic. (b) This image in a zoom of the bottom-left corner of Fig. 2. It shows in overlay the mosaic (in white) and the goedesic path (in black) to reconstruct it by "continuation". If some closure seem to correctly reconstruct the borders, some others appear to be at the wrong place.

Applied to two points laying on the cornea borders, the shortest path between those two points coincide with a border a of cell (Fig. 10a). It seems logic to wait for good results with this method. But, when trying to connect one extremity with the closest other extremity, the connection is not what is expected (Fig. 10b). These results mean that something more is used to visually infer the cells: in fact, some regional informations are necessary to first extract the extremities to be connected, and then to draw a path between them.

\section{REGION-BASED OF THE MOSAIC \\ RECONSTRUCTION}

More efficient methods exist to segment mosaic images like cornea cells. They go one step further into the process of reconstruction, using a regional information to make a good closure of the cells. For example, the methods proposed by Vincent and 
Masters (1992) and Angulo and Matou (2005) perform a really accurate detection and reconstruction of the mosaic in cornea endothelial. This subsection describes those methods and the explanation of their good results according to human visual principles previously presented.

These methods (Alg. 2, 3) follow the general algorithm (Alg. 1): after a filtering process (alternate sequential filtering or simply opening), both methods consist on extracting markers of the corneal cells and using them in a constrained watershed to get the borders of the cells. The difference is that Alg. 2 does an effective reconstruction whereas Alg. 3 directly performs a detection of closed contours.

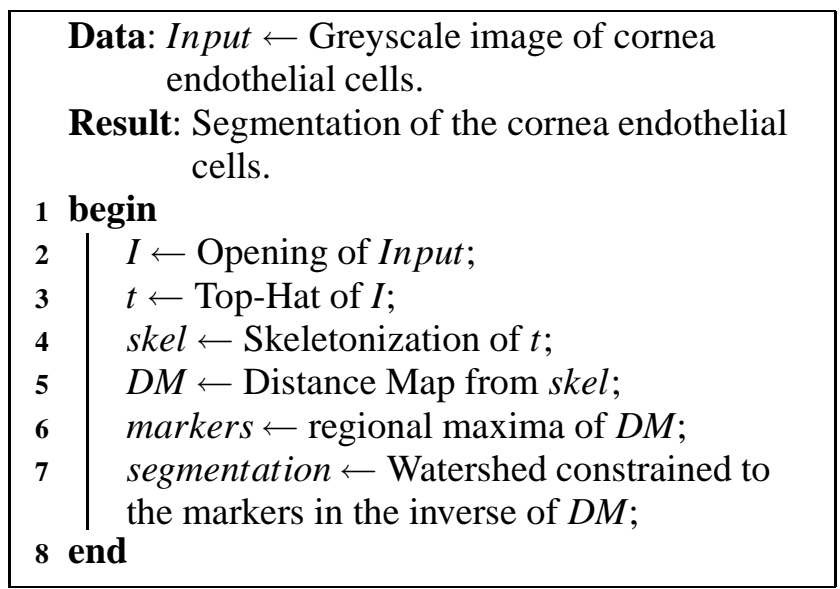

Algorithm 2: Angulo's method for detecting the cells (Angulo and Matou, 2005).

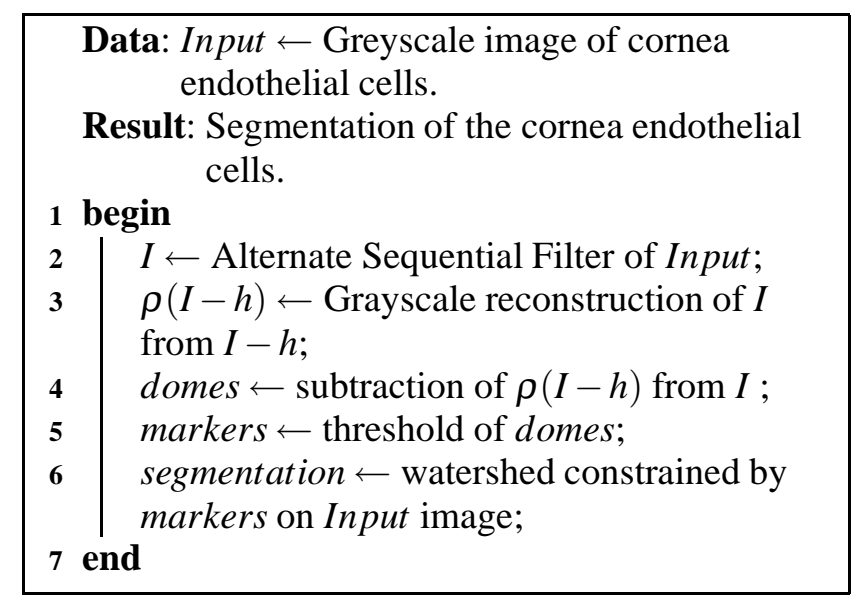

Algorithm 3: Vincent's method for detecting the cells (Vincent and Masters, 1992).

\section{SOME EXPLANATIONS ON THE ALGORITHMS}

These algorithms are based upon the mathematical morphology (Serra, 1982; Soille, 2003): erosion and dilation are the basic operators, and their combination enables the definition of opening and closing operators, top-hat operator (used to detect crests) and alternate sequential filters (alternance of opening and closing of growing sizes). The watershed is also based upon mathematical morphology. If a distance map is considered as a grey level image, it may be interpreted as a surface (the grey level is seen as an elevation). The watershed partitions such an image into catchment basins, each marker being a regional maximum of the distance map. As previously said, those basins are connected. The main problem using the watershed is to constrained them with relevant markers (hence the choice of the markers is crucial).

The distance map associates a distance value from every pixel in an image to an object. For example, the distance map from every pixel to the skeleton of the borders of the cells is represented in Fig. 11(c). When locating the maxima of this map, they can be roughly considered as the centers of the cell bodies and be used to constrain the watershed.

In those region-based methods, the distance criterion is introduced implicitely by detecting the centers of the cells with a distance map (Angulo and Matou, 2005) or with an extended regional maxima (extraction of $h$-domes in Vincent and Masters, 1992). Thus, the regional information that was missing in the cell borders detection methods is used to segment the cells, but there are still remaining problems.

The results of the segmentations can be observed in Fig. 12. This is almost what is visually expected, except that there is an over-segmentation. The noisy image does not simply provide the centers of the cells, and this is the main problem of these algorithms (Algs. 2, 3), due to non-relevant markers (Soille, 2003). One advantage of using a distance map to get the markers is that it is well adapted to detecting convex cells. For non convex cells this could generate errors (like grain boundaries in material sciences).

\section{IMPROVEMENTS IN CORNEAL CELLS DETECTION}

A more efficient method has been recently developped (Gavet, 2008). Its efficiency can be explained by a better detection of the regional markers: the supremum of openings by segments preserves the linear parts of the mosaic (Fig. 4). This is inspired from Chazallon and Pinoli (1997), where only the principal component of the skeleton is retained. 


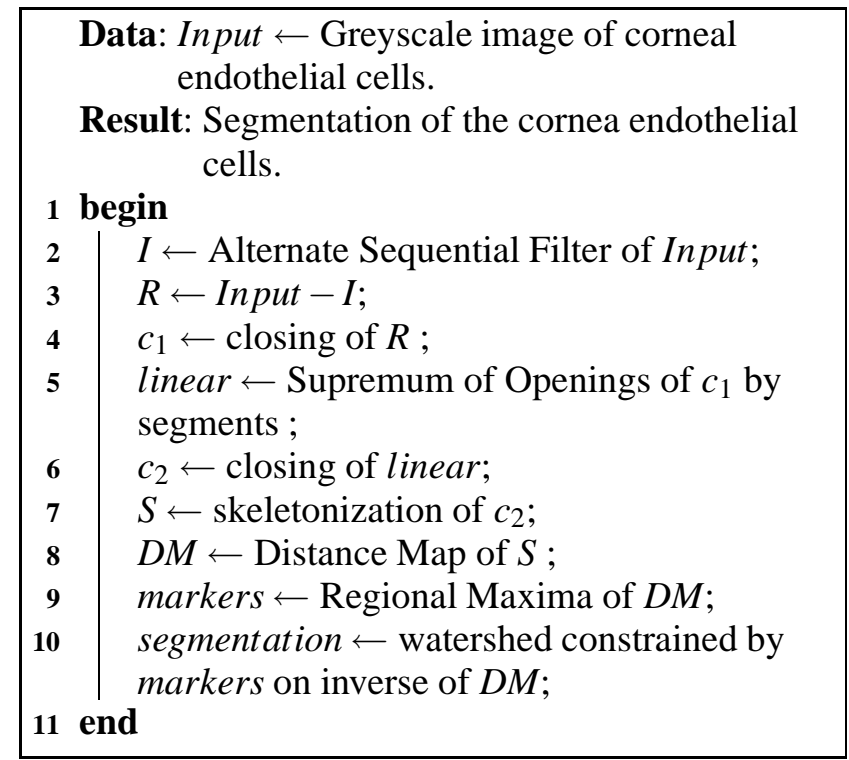

Algorithm 4: Present article's method for detecting the cornea endothelial cells.

The structure of this algorithm (Alg. 4) is similar to the one Alg. 1 (see Fig. 11); the reconstruction step is the same as for Angulo and Matou (2005), and the filter is an alternate sequential (as for Vincent and Masters, 1992). The main difference is the opening by segments (line 5) followed by a closing (line 6), that performs a filter (by keeping the linear parts) of the borders: the skeleton is thus more accurate, and the reconstruction is made easier.

This mosaic alone is already enough for a visual reconstruction by an expert (since the grey level information is not present, an observer has to know that certain types of cells, too litle or not convex, do not exist in a real cornea).

\section{RESULTS AND DISCUSSION}

Focusing only on the contours to extract a mosaic is not enough to perform a good segmentation. Some regional informations like markers pointing the centers of the cells improve the detection of the mosaic (see Fig. 12), but this is not perfect. This is due to a detection of too many markers, or in fact because of a detection of wrong borders.

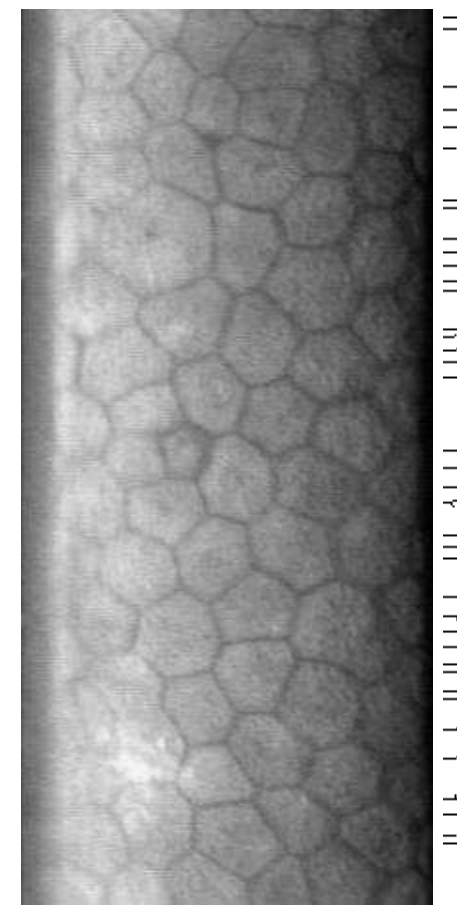

(a)

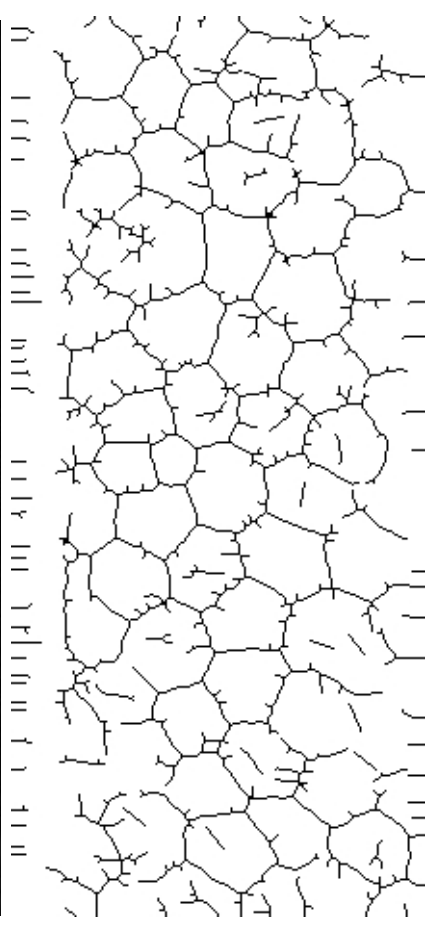

(b)

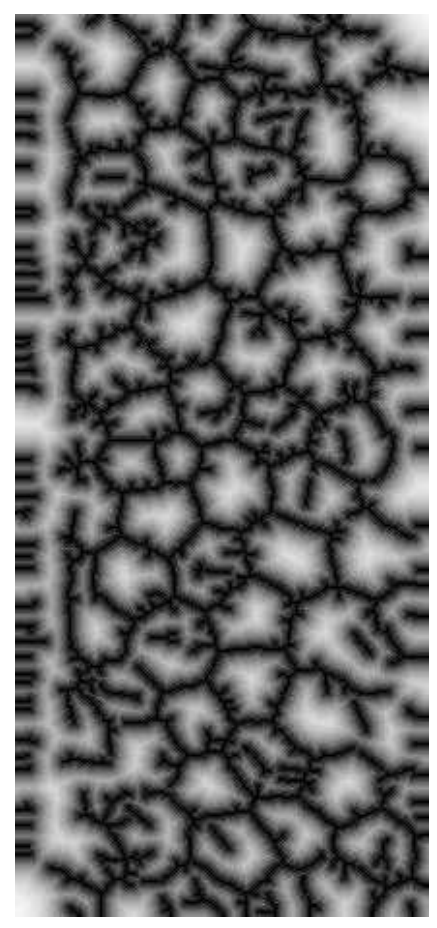

(c)

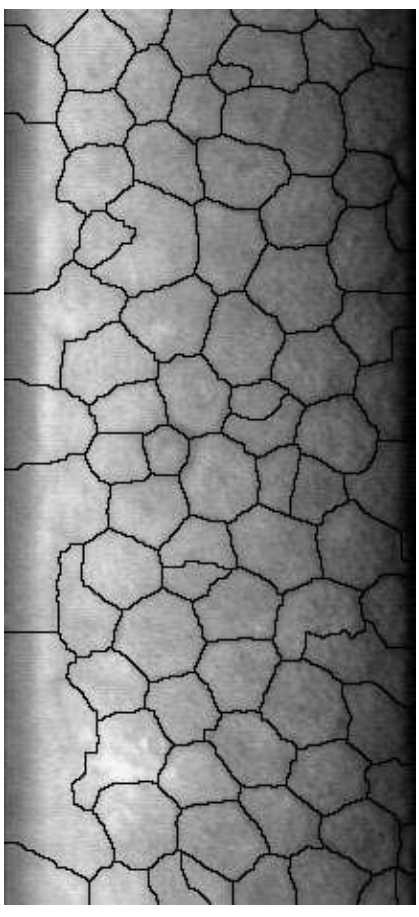

(d)

Fig. 11. Mosaic recognition within the image (a) using Alg. 4. (a) Specular microscopy image of a human corneal endothelium. (b) Corneal endothelium mosaic before reconstruction. This skeleton is the result at line 6 of Alg. 4. (c) Distance map from the skeleton presented in (b). Every pixel is given the value of the Euclidean distance to the original skeleton, and represented as an image. The local maxima in this image are used as markers for the watershed process. (d) Present article's resulting mosaic. 


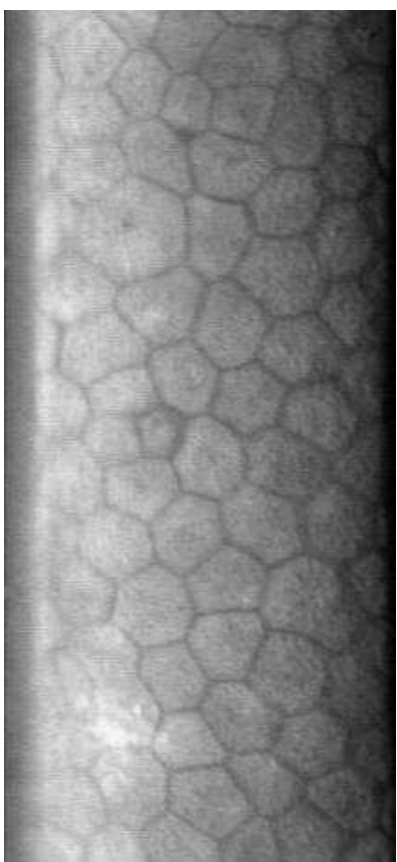

(a)

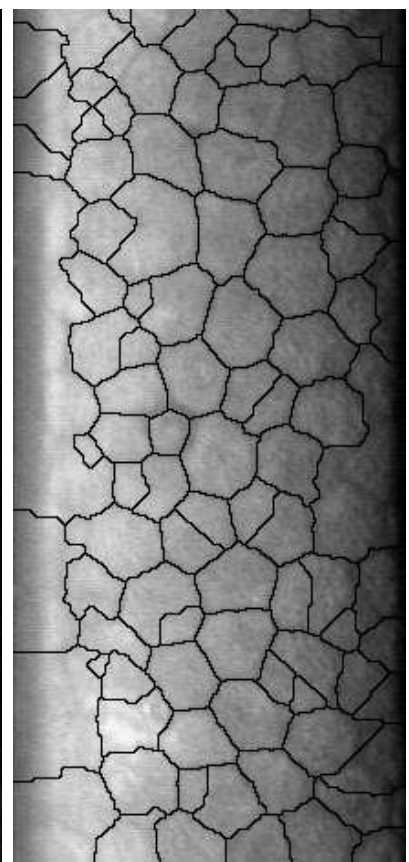

(b)

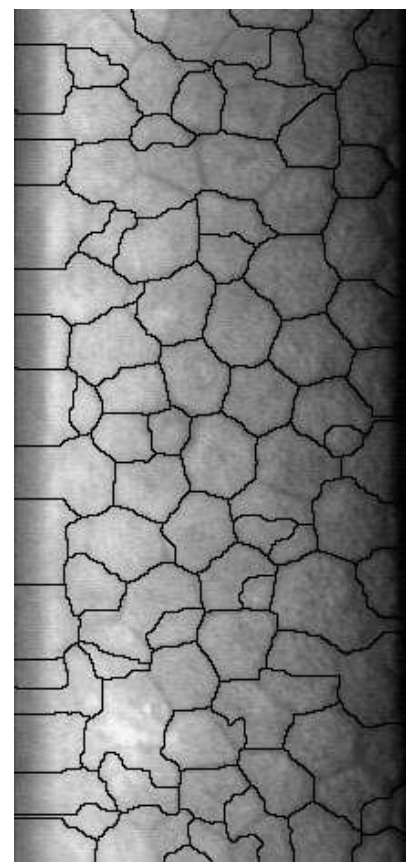

(c)

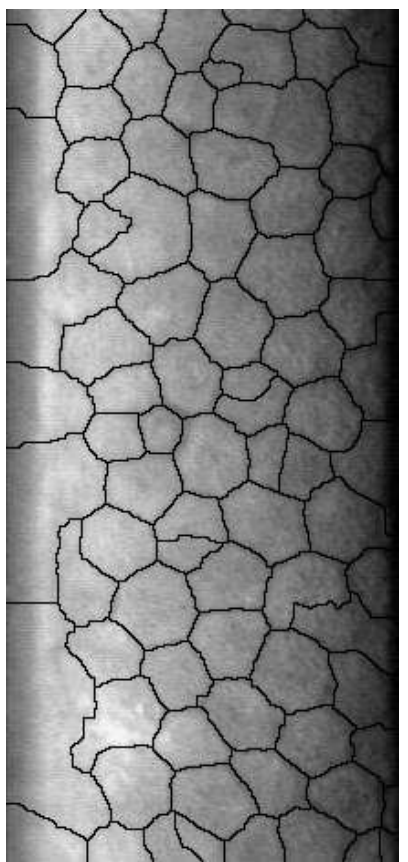

(d)

Fig. 12. Mosaic recognition within the image 12(a) by different methods. Excepted at the left and right sides of the images (where hardly nothing can be seen), the detection is really efficient, especially for the proposed method $(d)$. But there subsists some problems that remains visually evident. (a) Specular microscopy image of a human corneal endothelium. (b) Angulo's method (Angulo and Matou, 2005) applied on (a). (c) Vincent's method (Vincent and Masters, 1992) applied on (a). (d) Present article's resulting mosaic.

By using a supremum of openings by segments, the proposed algorithm (see Fig. 12d) eliminates a lot of non cell borders, but not all of them. In practice, there will always be cases where false borders will be detected. The next improvements of this algorithm will be placed on avoiding the detection of these false borders and/or on knowing that they are false (Gavet, 2008).

\section{CONCLUSION AND PERSPECTIVES}

Theories of visual perception provide explanations of the efficiency of some image analysis methods: their study allows to improve their use in some new algorithms, specially adapted to the endothelial mosaic structure. The presented method gives better results than previous ones. It makes a better use of the knowledge of the human perception and of the particular geometry of the corneal mosaics.

In a near future, this method will be improved by using this duality "region-contour": a contour detection followed by a region analysis to then reconstruct the mosaic by closing the contours.
This last step will be done by computing regional geometrical or morphological factors, like symmetry or shape criteria. Those parameters should be efficient to detect false positives, e.g., distinguish erroneously detected cells from real ones.

\section{ACKNOWLEDGEMENTS}

The authors wish to thank Prof. Philippe Gain and Dr. Gilles Thuret (CHU of Saint-Etienne, France) for their contribution. This work is a part of a $\mathrm{PhD}$ Thesis (Gavet, 2008) and also Gavet et al. (2007).

\section{REFERENCES}

Angulo J, Matou S (2005). Automatic quantification of in vitro endothelial cell networks using mathematical morphology. In: IASTED Int Conf Visualiz Imaging Image Proc (VIIP'2005). Benidorm, Spain: IASTED, 51-6.

Ayala G, Díaz ME, Martínez-Costa L (2001). Granulometric moments and corneal endothelium status. Pattern Recogn 34:1219-27.

Beucher S, Lantuejoul C (1979). Use of watersheds in contour detection. In: Int Worksh Image Proc. Rennes, France. 
Blum H, Nagel RN (1978). Shape description using weighted symmetric axis features. Pattern Recogn 10:167-80.

Calabi L, Hartnett W (1968). Shape recognition, prairie fires, convex deficiencies and skeletons. Am Math Mon 75:335-42.

Chazallon L, Pinoli JC (1997). An automatic morphological method for aluminium grain segmentation in complex grey level images. Acta Stereol 16:119-30.

Cuisenaire O (1999). Distance transformations: fast algorithms and applications to medical image processing. Ph.D. thesis, Université Catholique de Louvain.

Dakin SC, Hess RF (1999). Contour integration and scale combination processes in visual edge detection. Spatial Vision 12:209-327.

de Andrade MC (2004). An interactive algorithm for image smoothing and segmentation. Electron Lett Comput Vision Image Anal 4:32-48.

Debayle J, Gavet Y, Pinoli JC (2006). General adaptive neighborhood image restoration, enhancement and segmentation. In: Campilho A, Kamel M, eds., Proc Int Conf Image Anal Recogn (ICIAR), no. 4141 in Lecture Not Comput Sci. Springer Verlag, 29-40.

Doughty M, Spiteri M, Dilts D (1997). Determination of the unit size of the corneal endothelial cell mosaic from fourier component image analysis. Tissue Cell 29:22938.

Fitzke FW, Masters BR, Buckley RJ, Speedwell L (1997). Fourier transform analysis of human corneal endothelial specular photomicrographs. Exp Eye Res 65:205-14.

Foracchia M, Ruggeri A (2000). Cell contour detection in corneal endothelium in-vivo microscopy. Proc 22nd Ann Int Conf IEEE 2:1033-5.

Gain P, Thuret G, Kodjikian L, Gavet Y, Turc PH, Theillere C, Acquart S, Petit JCL, Maugery J, Campos L (2002). Automated tri-image analysis of stored corneal endothelium. Br J Ophthalmol 86:801-8.

Gavet Y (2008). Perception visuelle humaine, complétion des mosaïques et application à la reconstruction d'images de l'endothélium cornéen humain en microscopie optique spéculaire. Ph.D. thesis, École Nationale Supérieure des Mines de Saint-Étienne.

Gavet Y, Pinoli JC, Thuret G, Gain P (2007). Human visual perception, gestalt principles and duality regioncontour. Application to computer image analysis of human cornea endothelium. In: Ranchordas A, Araújo H, Vitrià J, eds., Proc 2nd Int Conf Comput Vision Theory App, Barcelona, 7-11 March, vol. 2. Setúbal, Portugal: INSTICC, 221-4.

Hateren JH, Srinivasan MV, Wait PB (1990). Pattern recognition in bees: orientation discrimination. J Comp
Physiol A V167:649-54.

$\mathrm{Hu}$ J, Yan H (1997). Polygonal approximation of digital curves based on the principles of perceptual organization. Pattern Recogn 30:701-18.

Ikonen L, Toivanen P (2005). Shortest routes on varying height surfaces using gray-level distance transforms. Image Vision Comput 23:133-41.

Kanizsa G (1980). Grammatica del vedere. Saggi su percezione e Gestalt. Il Mulino, Bologna.

Koffka K (1935). Principles of Gestalt Psychology. New York: Harcourt Brace.

Kovács I, Julesz B (1993). A closed curve is much more than an incomplete one: Effect of closure in figure-ground segmentation. Proc Natl Acad Sci USA 90:7495-7.

Kubovy M, Gepshtein S (2000). Gestalt: from phenomena to laws. In: Boyer KL, Sarkar S, eds., Perceptual organization for artificial vision systems. Dordrecht: Kluwer Academic Publishers, 41-71.

Marr D (1983). Vision: A computational investigation into the human representation and processing of visual information. New York: Henry Holt \& Company.

Mullen KT, Beaudot WHA, McIlhagga WH (2000). Contour integration in color vision: a common process for the blue-yellow, red-green and luminance mechanisms? Vision Res 40:639-55.

Nieder A (2002). Seeing more than meets the eye: processing of illusory contours in animals. J Comp Physiol A 188:249-60.

Rock I (2001). La Perception. Paris: De Boeck Université.

Salerno M, Sargeni F, Bonaiuto V, Amerini P, Cerulli L, Ricci F (1998). A new CNN based tool for an automated morphometry analysis of the corneal endothelium. In: Proc 5th IEEE Int Worksh Cell Neural Netw Appl, London, 14-17 April 1998. New York: IEEE, 169-74.

Serra J (1982). Image analysis and mathematical morphology. London: Academic Press.

Soille P (2003). Morphological image analysis: Principles and applications. New York: Springer.

Vincent L, Masters B (1992). Morphological image processing and network analysis of cornea endothelial cell images. In: Gader PD, Dougherty ER, Serra JC, eds., Image Algebra and Morphological Image Processing III, vol. 1769. San Diego, CA: SPIE, 21226.

Wertheimer M (1923a). Untersuchungen zur Lehre von der Gestalt. I. Psychol Forsch 1:47-58.

Wertheimer M (1923b). Untersuchungen zur Lehre von der Gestalt. II. Psychol Forsch 4:301-50.

Zou JJ, Chang HH, Yan H (2001). Shape skeletonization by identifying discrete local symmetries. Pattern Recogn 34:1895-905. 